\title{
Exogenous damage causes cell DNA damage through mediated reactive oxygen levels
}

\author{
Peiyan Guo ${ }^{1}$, Ning Ma ${ }^{1}$, Jingbo Shan ${ }^{1}$, Techang Chen ${ }^{1}$, Yujie Zhang ${ }^{1}$, Sa Zhou ${ }^{1, a}$ and Wenjian Ma ${ }^{1,2, a}$ \\ ${ }^{1}$ Key Laboratory of Industrial Microbiology, Ministry of Education and Tianjin City, College of Biotechnology, Tianjin University of \\ Science and Technology \\ ${ }^{2}$ Qilu Institute of Technology, Shandong 250200, PR China
}

\begin{abstract}
Many anti-tumor drugs can induce tumor apoptosis by increasing intracellular ROS. In the present study, we build a model which did not directly cause DNA damage, but simulated damage products. The model of this injury was transferred into the cell so that the cell's damage recognition mechanism mistakenly recognized that its own DNA was damaged, which in turn induced a response. Based on this model, the damaged plasmids (exogenous DNA damage) were transferred into the cells and the amount of reactive oxygen in the cells was improved, and DNA damage of the cells was increased. Therefore, exogenous DNA damage can affect the accumulation of damage in cells by affecting the level of reactive oxygen species, which provides a reference for DNA damage repair research.
\end{abstract}

\section{Introduction}

Exogenous injury refers to the treatment of oligonucleotides in vitro with drugs, resulting in various types of DNA damage. We disrupted oligonucleotides (plasmids) in three ways, cisplatin, oxaliplatin and PUVA. Cisplatin, the main target of DNA, which can cause DNA chain cross-linking, thereby inhibiting cell DNA replication, cisplatin is the first generation of platinum anti-cancer drugs [1-2]. Oxaliplatin is a thirdgeneration platinum compound, and Oxaliplatin's pharmacological properties are similar to other platinum drugs, targeting DNA. Oxaliplatin is easily combined covalently with $\mathrm{G}$ and may form an inter-chain crosslink, thus blocking DNA replication and transcription [3-5]. Psoralen is photosensitive, and under ultraviolet (UVA) exposure, react with DNA to form a type of DNA injury - inter-strand crosslink (ICL) [6-7]. The DNA containing the damage is transferred to the human cells, whether the response in the cell will be induced. In this paper, the response change of reactive oxygen was mainly explored.

Reactive oxygen species (ROS) is a single electron reduction product of oxygen in the body. It is the electron that leaks out of the respiratory chain before it is delivered to the terminal oxidase and consumes about $2 \%$ of oxygen. It has more oxygen than molecular oxygen.[8]. Under pathological conditions, the excessive production of ROS exceeds the endogenous antioxidant defense, oxidative stress may irreversibly modify (oxidizing) biological macromolecules, such as DNA, protein, carbohydrate and lipid [9], and the production of $\mathrm{O}_{2}$ can also lead to the loss of NO bioavailability [10]. However, in recent years, with the further research the biological function of ROS, the role of ROS anti-killer tumor cells has been gradually recognized, and the formation of a new free radical biomedical research field [11].

Oxidative stress may irreversibly modify (oxidize) biological macromolecule DNA, while DNA doublestrandbreak break (Double-strand break, DSB) is the most severe form of damage, and unrepairable DNA double-stranded fractures can cause cell death [12-13]. One of the earliest reactions after a double-stranded fracture of DNA was the phosphorylation of the C-end serine residue of the histone $\mathrm{H} 2 \mathrm{AX}$ near the fracture point, forming $\gamma$-H2AX [14], triggering a series of biocascades and cytological reactions [15-16]. By far, $\gamma-$ $\mathrm{H} 2 \mathrm{AX}$ is the most important DNA damage sensing molecule studied [17-18].

\section{Materials and Methods}

\subsection{Plasmid damage}

Cisplatin, oxaliplatin, dissolved in PBS buffer, and diluted into a series of solutions. 25 ul of pmCherry plasmid DNA was mixed with $75 \mu$ l of different concentrations of cisplatin and oxaliplatin, and incubated at $37^{\circ} \mathrm{C}$ for $2 \mathrm{~h}$ in a water bath. The psoralen $50 \mathrm{ng} / \mathrm{ml}$ was treated with pm-cherry plasmid for $16-18 \mathrm{~h}$, followed by UVA treatment for $6 \min 19 \mathrm{~s}$.

\subsection{Cell Culture}

MCF-7 cells were cultured in RPMI 1640 medium (Gibco, Invitogen). All media contained 10\% FBS (Sijiqing), $100 \mathrm{~g} / \mathrm{mL}$ penicillin and $100 \mathrm{~g} / \mathrm{mL}$ streptomycin. All cells were cultured in $37{ }^{\circ} \mathrm{C}, 5 \% \mathrm{CO} 2$

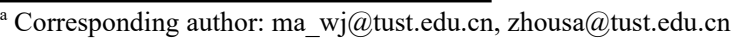


cell incubator.

\subsection{Western blot analysis}

Cells were collected and lysed for 30 minutes at $4^{\circ} \mathrm{C}$ in protein lysis buffer. Protein concentration was determined by BCA kit (Beyotime, China). The 30-50ug protein samples were separated by SDS-PAGE (12\%) gel, concentrated by $4 \%$, and semi-dried transferred to $\mathrm{NC}$ membrane. The membrane was sealed with 5\% skimmed milk for 1 hour and incubated overnight with specific primary antibody at $4^{\circ} \mathrm{C}$. Specific membranes were sealed for 2 hours with secondary antibodies. After washing, Odyssey infrared laser imaging system was used for detection. Beta-actin or GAPDH were used as internal parameters for protein level detection.

\subsection{Measurement of ROS}

ROS generation was measured using oxidation sensitive fluorescent probe (DCFH-DA) according to the manufacturer's protocols (Beyotime, China). Cells were treated with olaparib and rucaparib in the absence or presence of NAC and Apocynin for the indicated times. After the incubation, the cells were harvested and then stained with $10 \mu \mathrm{M}$ DCFH-DA probe at $37^{\circ} \mathrm{C}$ for 30 min. Cells were washed three times with PBS, and the induction of ROS was examined by Confocal fluorescence microscopy.

\subsection{Statistical analysis}

The software Graphpad Prism 7 was used for statistical analysis. All data were presented as means and standard errors. Student's t-test and oneway ANOVA analysis were used to determine significance. $\mathrm{P}<0.05$ was considered statistically significant. Statistical significance was also taken as $* \mathrm{P}<0.05, * * \mathrm{P}<0.01$ and $* * * \mathrm{P}<0.001$.

\section{Results}

\subsection{Establish a research model containing DNA damage plasmids using DNA-damaging drugs.}

The Pmcherry-CIBN-CreC plasmid DNA fragmentation experimental electropherogram was used to explore the DNA fragmentation of two platinum anticancer drugs (Fig. 1). The rate of supercoiled structure of the plasmid became slow at cisplatin at $0-200 \mu \mathrm{M}$ and oxaliplatin at $0-800 \mu \mathrm{M}$ in the treatment concentration range. It indicated that platinum compounds caused rupture damage to plasmid DNA, and also induced cross-linking between DNA single strands or double strands. These DNAs are subjected to migration resistance greater than that of intact supercoiled plasmid DNA. Since the manner and quantity of fracture and cross-linking are irregular, the configuration of each broken DNA molecule is different and the mobility is different. Psorale is a photosensitive substance that, under ultraviolet light (UVA), reacts with DNA to form a type of DNA injury interstitated cross-link (ICL). As shown in Figure 1, PUVA (Psoralen and UVA) processes plasmid DNA, causing the cross linking between plasmid chains, resulting in a slow electrophoresis.

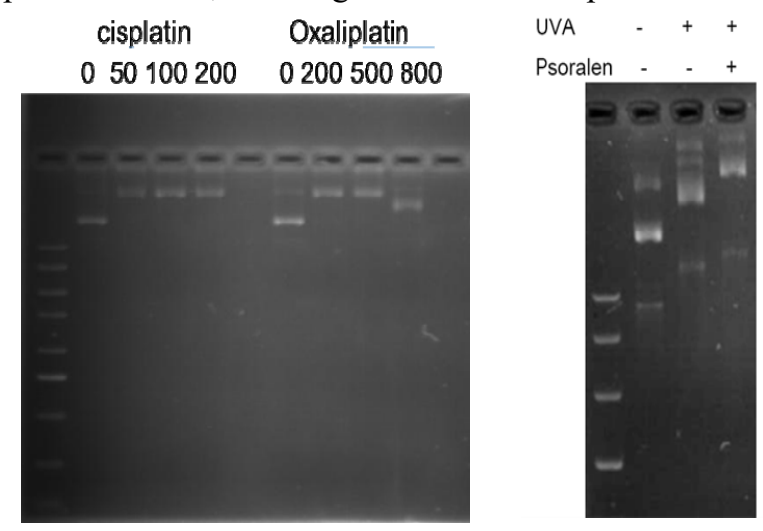

Figure 1. drug treatment plasmid electrophoresis map.

\subsection{Transferring DNA damage models into cells could cause changes in reactive oxygen species levels.}

The purified plasmid was quantified, and the cells were transfected according to the instructions of the transfection reagent, and the changes in reactive oxygen species in the cells were measured 6 hours later. The plasmid was treated with different concentrations of drug and the ROS produced by the cells were transfected (Fig. $2 a, 2 b, 2 c)$. Green represents the ROS produced by the cells, and red is the plamid color, indicating that the plasmid was successfully transferred into the cell, and the last column is the merge of ROS plasmid. The figure shows that exogenous DNA transfected cells cause an increase in the level of cellular reactive oxygen species. As the concentration of the drug increases, the efficiency of exogenous DNA transfer into the cell decreases (red portion in the figure).

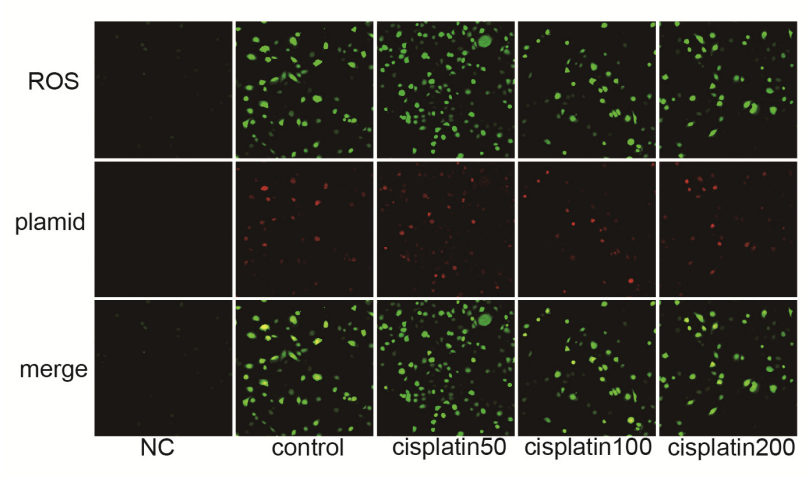

Figure 2a. The ROS produced by transfection cells is treated with different concentrations of cisplatin plasmids 


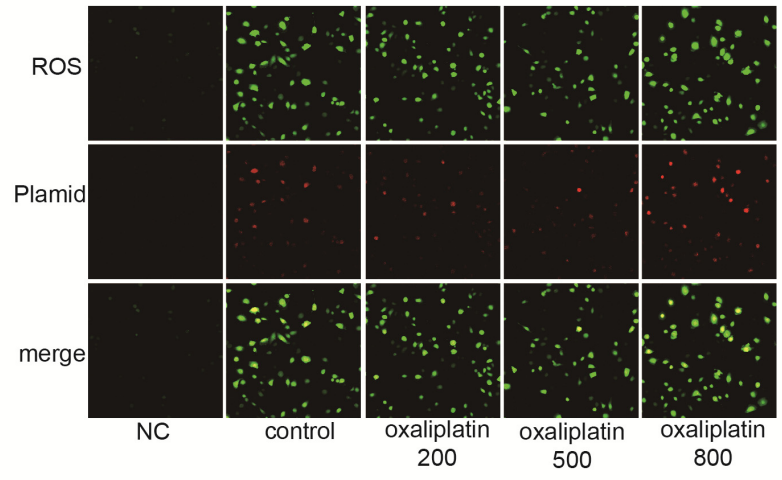

Figure $\mathbf{2 b}$. The ROS produced by transfection cells is treated with different concentrations of oxaliplatin to treat plasmids.

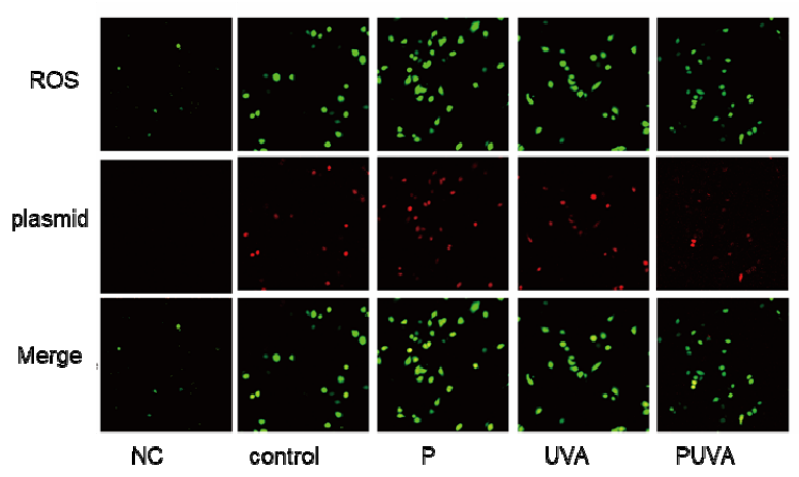

Figure 2c. The plasmids are treated with PUVA (Psoralen and UVA and PUVA) respectively, and the ROS produced after transfer to the cells.

\subsection{Effect of reactive oxygen species on cell DNA damage.}

The level of intracellular free radicals is an important factor affecting DNA damage. The formation of reactive oxygen species can leaded to DNA damage. We detected the expression of DNA damage marker $\gamma$-H2AX in the cells after drug treatment by western blot. As shown in Fig-3, the results showed that the exogenous DNA damage could induce the increase of the level of reactive oxygen species in the cells, and then cause the changes of cell DNA damage.

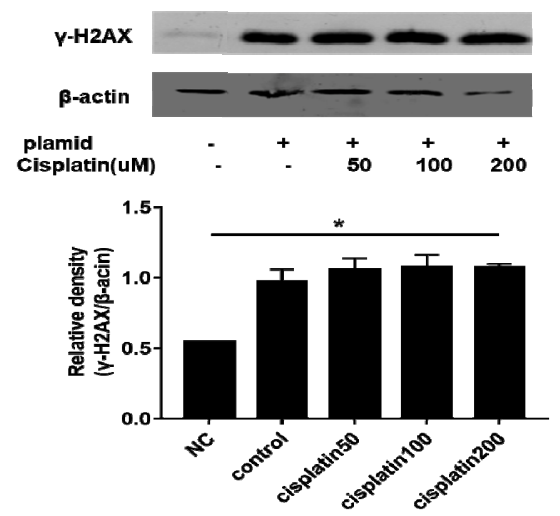

Figure 3a. Cisplatin-treated plasmid influence the DNA damage of recipient cells.

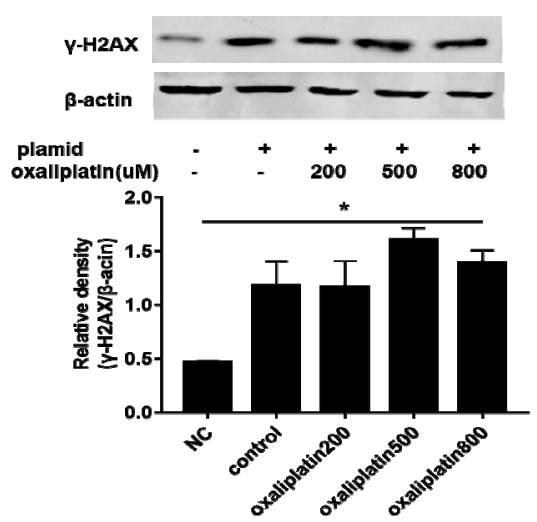

Figure 3b. oxaliplatin-treated plasmid influence the DNA damage of recipient cells.

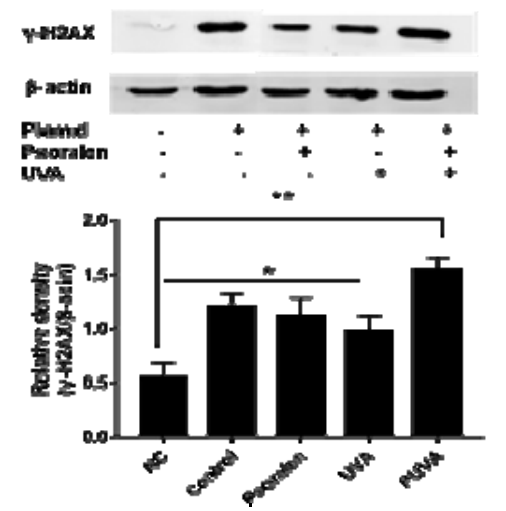

Figure 3c. PUVA-treated plasmid influence the DNA damage of recipient cells.

\subsection{A diagram of the research ideas in this paper.}

Cell metabolic activities and external radiation to cells can produce a series of reactive oxygen species, which are necessary for certain physiological processes of cells. However, higher levels of reactive oxygen species could have harmful effects on cells by affecting macromolecules such as DNA and proteins. For example, hydroxyl radicals in reactive oxygen species can directly interact with DNA and destroy the skeleton structure of bases and DNA. Therefore, in this paper, a kind of exogenous damage DNA transferred into the cell, could also cause changes in the cellular microenvironment, resulting in DNA damage, and even cell death.

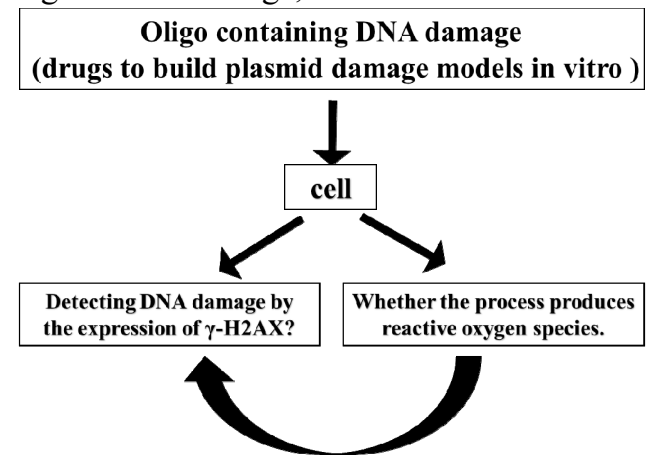

Figure 4. Experimental idea diagram. 


\section{Conclusions}

In this study, we found that exogenous damage DNA could cause a slight increase in the level of intracellular reactive oxygen species, which led to the increase of DNA damage. Therefore, in research involving reactive oxygen species and DNA damage, it is necessary to consider exogenous DNA causes cellular DNA damage by affecting reactive oxygen species levels. As for how the exogenous DNA caused the change of the level of reactive oxygen species, we speculate that it may be related to the change of the extracellular microenvironment caused by the exogenous DNA damage, and the specific mechanism needs to be further studied.

\section{Conflict of Interest}

We have no conflicts of interest to disclose.

\section{References}

1. M. Alomari, B. R. Jermy, V. Ravinayagam, S. Akhtar, S. A. Almofty, S. Rehman, H. Bahmdan, S. AbdulAzeez, J. F. Borgio, Cisplatin-functionalized three-dimensional magnetic SBA-16 for treating breast cancer cells (MCF-7). Artificial Cells, Nanomedicine, and Biotechnology. 47, 3079-3086 (2019).

2. M. Tsunematsu, K. Haruki, T. Sakamoto, T. Uwagawa, H. Shiba, K. Yanaga, Radical resection of an initially unresectable intrahepatic cholangiocarcinoma after chemotherapy with using gemcitabine, cisplatin, and S-1: report of a case. surg case rep. 5, 103 (2019).

3. S. Yamamoto, T. Yamashita, M. Ito, J. M. M. Caaveiro, N. Egashira, H. Tozaki-Saitoh, M. Tsuda, New pharmacological effect of fulvestrant to prevent oxaliplatin-induced neurodegeneration and mechanical allodynia in rats. Int. J. Cancer. 145, 2107-2113 (2019).

4. H. Liang, Y. Xu, Q. Zhang, Y. Yang, Y. Mou, Y. Gao, R. Chen, C. Chen, P. Dai, MiR-483-3p regulates oxaliplatin resistance by targeting FAM171B in human colorectal cancer cells. Artificial Cells, Nanomedicine, and Biotechnology. 47, 725-736 (2019).

5. A. Šebestová, D. Baron, R. Pechancová, T. Pluháček, J. Petr, Determination of oxaliplatin enantiomers at attomolar levels by capillary electrophoresis connected with inductively coupled plasma mass spectrometry. Talanta. 205, 120151 (2019).

6. C. Coppard, D. Hannani, M. Humbert, V. Gauthier, J. Plumas, E. Merlin, F. Gabert, L. Chaperot, In vitro PUVA treatment triggers calreticulin exposition and HMGB1 release by dying $\mathrm{T}$ lymphocytes in GVHD: New insights in extracorporeal photopheresis. J Clin Apher. 34, 450-460 (2019).
7. L. Yousefkhani, R. Rafiei, A. Darjani, N. Alizadeh, H. Eftekhari, K. Gharaei Nejad, E. Rafiei, B. Rafiee, Evaluation of serum folate level before and after bath PUVA therapy in patients referred to Razi Hospital, Rasht, Iran. Dermatologic Therapy (2019).

8. M. Valko, D. Leibfritz, J. Moncol, M. T. D. Cronin, M. Mazur, J. Telser, Free radicals and antioxidants in normal physiological functions and human disease. Int. J. Biochem. Cell Biol. 39, 44-84 (2007).

9. H. Nohl, L. Gille, A. Kozlov, K. Staniek, Are mitochondria a spontaneous and permanent source of reactive oxygen species? Redox Rep. 8, 135-141 (2003).

10. M. Inoue, E. F. Sato, M. Nishikawa, A. M. Park, Y. Kira, I. Imada, K. Utsumi, Mitochondrial generation of reactive oxygen species and its role in aerobic life. Curr. Med. Chem. 10, 2495-2505 (2003).

11. L. Guo, C. Zhang, G. Chen, M. Wu, W. Liu, C. Ding, Q. Dong, E. Fan, Q. Liu, Reactive oxygen species inhibit biofilm formation of Listeria monocytogenes. Microbial Pathogenesis. 127, 183189 (2019).

12. A. Ciccia, S. J. Elledge, The DNA damage response: making it safe to play with knives. Mol. Cell. 40, 179-204 (2010).

13. E. H. Choi, K. P. Kim, E2F1 facilitates DNA break repair by localizing to break sites and enhancing the expression of homologous recombination factors. Exp. Mol. Med. 51, 106 (2019).

14. A. Okabe, Y. Kiriyama, S. Suzuki, K. Sakurai, A. Teramoto, H. Kato, A. Naiki-Ito, S. Tahara, S. Takahashi, M. Kuroda, A. Sugioka, T. Tsukamoto, Short-term detection of gastric genotoxicity using the DNA double-strand break marker $\gamma$-H2AX. $J$ Toxicol Pathol. 32, 91-99 (2019).

15. S. Zhang, S. Hao, Z. Qiu, Y. Wang, Y. Zhao, Y. Li, W. Gao, Y. Wu, C. Liu, X. Xu, H. Wang, Cadmium disrupts the DNA damage response by destabilizing RNF168. Food and Chemical Toxicology. 133, 110745 (2019).

16. M. Majidinia, A. Bishayee, B. Yousefi, Polyphenols: Major regulators of key components of DNA damage response in cancer. DNA Repair. 82, 102679 (2019).

17. B. Wang, Z. Zhang, S. Xia, M. Jiang, Y. Wang, Expression of $\gamma-\mathrm{H} 2 \mathrm{AX}$ and patient prognosis in breast cancer cohort. J Cell Biochem. 120, 12958 12965 (2019).

18. Y. Lee, Q. Wang, I. Shuryak, D. J. Brenner, H. C. Turner, Development of a high-throughput $\gamma$-H2AX assay based on imaging flow cytometry. Radiat Oncol. 14, 150 (2019). 